\title{
APPROXIMATE BOUNDARY LAYER SOLUTION OF A MOVING BEAM PROBLEM
}

\author{
M. Pakdemirli and E. Özkaya \\ Department of Mechanical Engineering \\ Celal Bayar University 45140 \\ Muradiye, Manisa, Turkey
}

\begin{abstract}
The transverse vibrations of a simply supported beam moving with constant velocity is considered. The case of transition from string to beam effects is treated. In this model, the fourth order spatial derivative multiplies a small parameter and hence leads to a boundary layer problem. The problem is solved approximately using the method of multiple scales.
\end{abstract}

\section{INTRODUCTION}

Band saws, fiber textiles, paper sheets, aerial cable tramways, oil pipelines, magnetic tapes, power transmission belts are all classified as axially moving material problems. These problems are categorized into two groups: 1) axially moving strings 2) axially moving beams. Due to their technological importance, a vast amount of literature exists on the topic. The review papers by Ulsoy et al. [1] and Wickert and Mote [2] contains work on axially moving strings and beams upto 1988.

For more recent work on beam vibrations see Wickert and Mote [3], Wickert [4], Pellicano and Zirilli [5] a j Öz et al. [6]. The transition behavior from string to beam has first been investigated by references [5] and [6]. In reference [5], a boundary layer solution was presented using a modified Lindstedt Poincare method. In [6], an outer solution was presented using the method of multiple scales. A stability analysis was also included.

Classical methods find the inner solution and outer solution separately and match the two solutions using physical constraints. The final solution is a composite expansion including the inner and outer solutions. On the other hand, using the method of multiple scales, the composite expansion can be retrieved at once using a single expansion [7]. In this work, the method of multiple scales is first applied to the small-stiffness axiallymoving-beam problem. The approximate solution is constructed. 


\section{THE METHOD OF MULTIPLE SCALES}

The linear dimensionless equation of motion for an axially moving beam with small flexural stiffness is [4]

$$
\frac{\partial^{2} y}{\partial t^{2}}+2 v \frac{\partial^{2} y}{\partial x \partial t}+\left(v^{2}-1\right) \frac{\partial^{2} y}{\partial x^{2}}+\varepsilon v_{f}^{2} \frac{\partial^{4} y}{\partial x^{4}}=0
$$

where $y(x, t)$ is the transverse displacement, $v$ is the constant velocity and $v_{f}^{2}$ is a dimensionless parameter [4]. A small parameter $\varepsilon$ is introduced to represent small beam effects. $\mathrm{x}$ and $\mathrm{t}$ are the dimensionless spatial and time coordinates respectively. The simply supported boundary conditions for the problem are

$$
y(0, t)=y(1, t)=0 \quad y^{\prime \prime}(0, t)=y^{\prime \prime}(1, t)=0
$$

Since the highest order derivative multiplies a small parameter, the problem is a boundary layer problem.

To apply the method of multiple scales, one defines the variables

$$
\mathrm{x}_{0}=\mathrm{x}, \quad \mathrm{x}_{1}=\frac{\mathrm{x}}{\sqrt{\varepsilon}}, \quad \mathrm{T}_{0}=\mathrm{t}, \quad \mathrm{T}_{1}=\varepsilon \mathrm{t}
$$

where $x_{0}$ is the outer variable and $x_{1}$ is the inner variable for the left boundary. $T_{0}$ is the usual fast time scale and $T_{1}$ is the slow time scale. The derivatives are defined as follows

$$
\begin{aligned}
& \frac{\partial}{\partial \mathrm{x}}=\frac{\partial}{\partial \mathrm{x}_{0}}+\frac{1}{\sqrt{\varepsilon}} \frac{\partial}{\partial \mathrm{x}_{1}} \\
& \frac{\partial^{2}}{\partial \mathrm{x}^{2}}=\frac{\partial^{2}}{\partial \mathrm{x}_{0}{ }^{2}}+\frac{2}{\sqrt{\varepsilon}} \frac{\partial^{2}}{\partial \mathrm{x}_{0} \partial \mathrm{x}_{1}}+\frac{1}{\varepsilon} \frac{\partial^{2}}{\partial \mathrm{x}_{1}{ }^{2}} \\
& \frac{\partial^{4}}{\partial \mathrm{x}^{4}}=\frac{\partial^{4}}{\partial \mathrm{x}_{0}{ }^{4}}+\frac{4}{\sqrt{\varepsilon}} \frac{\partial^{4}}{\partial \mathrm{x}_{0}{ }^{3} \partial \mathrm{x}_{1}}+\frac{6}{\varepsilon} \frac{\partial^{4}}{\partial \mathrm{x}_{0}{ }^{2} \partial \mathrm{x}_{1}{ }^{2}}+\frac{4}{\varepsilon \sqrt{\varepsilon}} \frac{\partial^{4}}{\partial \mathrm{x}_{0} \partial \mathrm{x}_{1}{ }^{3}}+\frac{1}{\varepsilon^{2}} \frac{\partial^{4}}{\partial \mathrm{x}_{1}{ }^{4}} \\
& \frac{\partial}{\partial \mathrm{t}}=\frac{\partial}{\partial \mathrm{T}_{0}}+\varepsilon \frac{\partial}{\partial \mathrm{T}_{1}} \\
& \frac{\partial^{2}}{\partial \mathrm{t}^{2}}=\frac{\partial^{2}}{\partial \mathrm{T}_{0}{ }^{2}}+2 \varepsilon \frac{\partial^{2}}{\partial \mathrm{T}_{0} \partial \mathrm{T}_{1}}
\end{aligned}
$$

Substituting the derivatives into the original equation, one has 
$\frac{\partial^{2} \mathrm{y}}{\partial \mathrm{T}_{0}^{2}}+2 \varepsilon \frac{\partial^{2} \mathrm{y}}{\partial \mathrm{T}_{0} \partial \mathrm{T}_{1}}+2 \mathrm{v}\left(\frac{\partial^{2} \mathrm{y}}{\partial \mathrm{x}_{0} \partial \mathrm{T}_{0}}+\varepsilon \frac{\partial^{2} \mathrm{y}}{\partial \mathrm{x}_{0} \partial \mathrm{T}_{1}}+\frac{1}{\sqrt{\varepsilon}} \frac{\partial^{2} \mathrm{y}}{\partial \dot{\mathrm{x}}_{1} \partial \mathrm{T}_{0}}+\sqrt{\varepsilon} \frac{\partial^{2} \mathrm{y}}{\partial \mathrm{x}_{1} \partial \mathrm{T}_{1}}\right)$

$+\left(v^{2}-1\right)\left(\frac{\partial^{2} y}{\partial x_{0}^{2}}+\frac{2}{\sqrt{\varepsilon}} \frac{\partial^{2} y}{\partial x_{0} \partial x_{1}}+\frac{1}{\varepsilon} \frac{\partial^{2} y}{\partial x_{1}^{2}}\right)$

$+\varepsilon \mathrm{v}_{\mathrm{f}}^{2}\left(\frac{\partial^{4} \mathrm{y}}{\partial \mathrm{x}_{0}{ }^{4}}+\frac{4}{\sqrt{\varepsilon}} \frac{\partial^{4} \mathrm{y}}{\partial \mathrm{x}_{0}{ }^{3} \partial \mathrm{x}_{1}}+\frac{6}{\varepsilon} \frac{\partial^{4} \mathrm{y}}{\partial \mathrm{x}_{0}{ }^{2} \partial \mathrm{x}_{1}{ }^{2}}+\frac{4}{\varepsilon \sqrt{\varepsilon}} \frac{\partial^{4} \mathrm{y}}{\partial \mathrm{x}_{0} \partial \mathrm{x}_{1}{ }^{3}}+\frac{1}{\varepsilon^{2}} \frac{\partial^{4} \mathrm{y}}{\partial \mathrm{x}_{1}{ }^{4}}\right)=0$

A suitable expansion for $\mathrm{y}(\mathrm{x}, \mathrm{t})$ would be

$\mathrm{y}(\mathrm{x}, \mathrm{t} ; \varepsilon)=\mathrm{y}_{0}+\sqrt{\varepsilon} \mathrm{y}_{1}+\varepsilon \mathrm{y}_{2}+\varepsilon \sqrt{\varepsilon} \mathrm{y}_{3}+\varepsilon^{2} \mathrm{y}_{4}+\ldots \ldots$

where $\mathrm{y}_{\mathrm{i}}$ all depend on $\mathrm{x}_{0}, \mathrm{x}_{1}, \mathrm{~T}_{0}$ and $\mathrm{T}_{1}$

equations

Substituting (6) into (5) and separating each order of $\varepsilon$, one obtains the set of

$\mathrm{O}\left(\frac{1}{\varepsilon}\right): \quad\left(\mathrm{v}^{2}-1\right) \frac{\partial^{2} \mathrm{y}_{0}}{\partial \mathrm{x}_{1}{ }^{2}}+\mathrm{v}_{\mathrm{f}}{ }^{2} \frac{\partial^{4} \mathrm{y}_{0}}{\partial \mathrm{x}_{1}{ }^{4}}=0$

$\mathrm{O}\left(\frac{1}{\sqrt{\varepsilon}}\right):\left(\mathrm{v}^{2}-1\right) \frac{\partial^{2} \mathrm{y}_{1}}{\partial \mathrm{x}_{1}{ }^{2}}+\mathrm{v}_{\mathrm{f}}{ }^{2} \frac{\partial^{4} \mathrm{y}_{1}}{\partial \mathrm{x}_{1}{ }^{4}}=-2 \mathrm{v} \frac{\partial^{2} \mathrm{y}_{0}}{\partial \mathrm{x}_{1} \partial \mathrm{T}_{0}}-2\left(\mathrm{v}^{2}-1\right) \frac{\partial^{2} \mathrm{y}_{0}}{\partial \mathrm{x}_{0} \partial \mathrm{x}_{1}}-4 \dot{\mathrm{v}}_{\mathrm{f}}{ }^{2} \frac{\partial^{4} \mathrm{y}_{0}}{\partial \mathrm{x}_{0} \partial \mathrm{x}_{1}{ }^{3}}$

$\mathrm{O}(1): \quad\left(\mathrm{v}^{2}-1\right) \frac{\partial^{2} \mathrm{y}_{2}}{\partial \mathrm{x}_{1}{ }^{2}}+\mathrm{v}_{\mathrm{f}}{ }^{2} \frac{\partial^{4} \mathrm{y}_{2}}{\partial \mathrm{x}_{1}{ }^{4}}=-\frac{\partial^{2} \mathrm{y}_{0}}{\partial \mathrm{T}_{0}{ }^{2}}-2 \mathrm{v} \frac{\partial^{2} \mathrm{y}_{0}}{\partial \mathrm{x}_{0} \partial \mathrm{T}_{0}}-2 \mathrm{v} \frac{\partial^{2} \mathrm{y}_{1}}{\partial \mathrm{x}_{1} \partial \mathrm{T}_{0}}-\left(\mathrm{v}^{2}-1\right)\left(\frac{\partial^{2} \mathrm{y}_{0}}{\partial \mathrm{x}_{0}{ }^{2}}\right.$

$$
\left.+2 \frac{\partial^{2} \mathrm{y}_{1}}{\partial \mathrm{x}_{0} \partial \mathrm{x}_{1}}\right)-\mathrm{v}_{\mathrm{f}}{ }^{2}\left(6 \frac{\partial^{4} \mathrm{y}_{0}}{\partial \mathrm{x}_{0}{ }^{2} \partial \mathrm{x}_{1}{ }^{2}}+4 \frac{\partial^{4} \mathrm{y}_{1}}{\partial \mathrm{x}_{0} \partial \mathrm{x}_{1}{ }^{3}}\right)
$$

$\mathrm{O}(\sqrt{\varepsilon}):\left(\mathrm{v}^{2}-1\right) \frac{\partial^{2} \mathrm{y}_{3}}{\partial \mathrm{x}_{1}{ }^{2}}+\mathrm{v}_{\mathrm{f}}{ }^{2} \frac{\partial^{4} \mathrm{y}_{3}}{\partial \mathrm{x}_{1}{ }^{4}}=-\frac{\partial^{2} \mathrm{y}_{1}}{\partial \mathrm{T}_{0}{ }^{2}}-2 \mathrm{v}\left(\frac{\partial^{2} \mathrm{y}_{1}}{\partial \mathrm{x}_{0} \partial \mathrm{T}_{0}}+\frac{\partial^{2} \mathrm{y}_{2}}{\partial \mathrm{x}_{1} \partial \mathrm{T}_{0}}+\frac{\partial^{2} \mathrm{y}_{0}}{\partial \mathrm{x}_{1} \partial \mathrm{T}_{1}}\right)$

$$
\begin{gathered}
-\left(v^{2}-1\right)\left(\frac{\partial^{2} y_{1}}{\partial x_{0}{ }^{2}}+2 \frac{\partial^{2} y_{2}}{\partial x_{0} \partial x_{1}}\right)-v_{f}{ }^{2}\left(4 \frac{\partial^{4} y_{0}}{\partial x_{0}{ }^{3} \partial x_{1}}\right. \\
\left.+6 \frac{\partial^{4} y_{1}}{\partial x_{0}{ }^{2} \partial x_{1}{ }^{2}}+4 \frac{\partial^{4} y_{2}}{\partial x_{0} \partial x_{1}{ }^{3}}\right)
\end{gathered}
$$


$O(\varepsilon):\left(v^{2}-1\right) \frac{\partial^{2} y_{4}}{\partial x_{1}{ }^{2}}+v_{f}{ }^{2} \frac{\partial^{4} y_{4}}{\partial x_{1}{ }^{4}}=-\frac{\partial^{2} y_{2}}{\partial T_{0}{ }^{2}}-2 \frac{\partial^{2} y_{01}}{\partial T_{0} \partial T_{1}}-2 v\left(\frac{\partial^{2} y_{2}}{\partial x_{11} T_{0}}+\frac{\partial^{2} y_{3}}{\partial x_{1} \partial T_{1}}\right.$

$$
\begin{gathered}
\left.+\frac{\partial^{2} \mathrm{y}_{0}}{\partial \mathrm{x}_{0} \partial \mathrm{T}_{1}}+\frac{\partial^{2} \mathrm{y}_{1}}{\partial \mathrm{x}_{1} \partial \mathrm{T}_{1}}\right)-\left(\mathrm{v}^{2}-1\right)\left(\frac{\partial^{2} \mathrm{y}_{2}}{\partial \mathrm{x}_{0}{ }^{2}}+2 \frac{\partial^{2} \mathrm{y}_{3}}{\partial \mathrm{x}_{0} \partial \mathrm{x}_{1}}\right) \\
-\mathrm{v}_{\mathrm{f}}{ }^{2}\left(\frac{\partial^{4} \mathrm{y}_{0}}{\partial \mathrm{x}_{0}{ }^{4}}+4 \frac{\partial^{4} \mathrm{y}_{1}}{\partial \mathrm{x}_{0}{ }^{3} \partial \mathrm{x}_{1}}+6 \frac{\partial^{4} \mathrm{y}_{2}}{\partial \mathrm{x}_{0}{ }^{2} \partial \mathrm{x}_{1}{ }^{2}}+4 \frac{\partial^{4} \mathrm{y}_{3}}{\partial \mathrm{x}_{0} \partial \mathrm{x}_{1}{ }^{3}}\right)
\end{gathered}
$$

\section{SOLUTIONS}

In this section, one solves the equations consequtively. At order $(1 / \varepsilon)$, the general solution is

$y_{0}=A\left(x_{0}, T_{0}, T_{1}\right) e^{\frac{\sqrt{1-v^{2}}}{v_{f}} x_{1}}+B\left(x_{0}, T_{0}, T_{1}\right) e^{-\frac{\sqrt{1-v^{2}}}{v_{f}} x_{1}}+C\left(x_{0}, T_{0}, T_{1}\right) x_{1}+D\left(x_{0}, T_{0}, T_{1}\right)$

For finite solutions, one chooses $\mathrm{A}=\mathrm{C}=0$. The term with $\mathrm{B}$ is a part of the inner solution. One may require $\mathrm{B}=0$ for not allowing the inner solution to appear at the first order. Hence

$$
\mathrm{y}_{0}=\mathrm{D}\left(\mathrm{x}_{0}, \mathrm{~T}_{0}, \mathrm{~T}_{1}\right)
$$

is the solution at this order.

For order $(1 / \sqrt{\varepsilon})$, one substitutes (13) into (8)

$$
\left(v^{2}-1\right) \frac{\partial^{2} y_{1}}{\partial x_{1}^{2}}+v_{f}^{2} \frac{\partial^{4} y_{1}}{\partial x_{1}^{4}}=0
$$

One may now choose

$$
\mathrm{y}_{1}=0
$$

At order (1), inserting (13) and (15) into (9) one obtains

$$
\left(v^{2}-1\right) \frac{\partial^{2} y_{2}}{\partial x_{1}{ }^{2}}+v_{f}{ }^{2} \frac{\partial^{4} y_{2}}{\partial x_{1}{ }^{4}}=-\frac{\partial^{2} D}{\partial T_{0}{ }^{2}}-2 v \frac{r^{2} D}{\partial x_{01} \partial T_{0}}-\left(v^{2}-1\right) \frac{\partial^{2} D}{\partial x_{0}{ }^{2}}
$$

In order not to introduce secular terms, D should be selected such that 


$$
\frac{\partial^{2} \mathrm{D}}{\partial \mathrm{T}_{0}^{2}}+2 \mathrm{v} \frac{\partial^{2} \mathrm{D}}{\partial \mathrm{x}_{0} \partial \mathrm{T}_{0}}+\left(\mathrm{v}^{2}-1\right) \frac{\partial^{2} \mathrm{D}}{\partial \mathrm{x}_{0}^{2}}=0
$$

Note that this equation is the equation for a constantly moving strip. A solution for $\mathrm{y}_{2}$ would then be

$$
y_{2}=E_{1}\left(x_{0}, T_{0}, T_{1}\right) e^{-\frac{\sqrt{1-v^{2}}}{v_{f}} x_{1}}+F_{1}\left(x_{0}, T_{0}, T_{1}\right)
$$

In above, only the decaying solutions are included.

At order $(\sqrt{\varepsilon})$, the solvability condition is

$$
\mathrm{v} \frac{\partial \mathrm{E}_{1}}{\partial \mathrm{T}_{0}}+\left(1-\mathrm{v}^{2}\right) \frac{\partial \mathrm{E}_{1}}{\partial \mathrm{x}_{0}}=0
$$

and $\mathrm{y}_{3}$ could be selected as

$$
\mathrm{y}_{3}=\mathrm{G}_{1}\left(\mathrm{x}_{0}, \mathrm{~T}_{0}, \mathrm{~T}_{1}\right) \mathrm{e}^{-\frac{\sqrt{1-\mathrm{v}^{2}}}{\mathrm{v}_{\mathrm{f}}} \mathrm{x}_{1}}
$$

Finally at order $(\varepsilon)$, one only determines the solvability conditions

$$
\begin{gathered}
\frac{\partial^{2} \mathrm{~F}_{1}}{\partial \mathrm{T}_{0}{ }^{2}}+2 \mathrm{v} \frac{\partial^{2} \mathrm{~F}_{1}}{\partial \mathrm{x}_{0} \partial \mathrm{T}_{0}}+\left(\mathrm{v}^{2}-1\right) \frac{\partial^{2} \mathrm{~F}_{1}}{\partial \mathrm{x}_{0}{ }^{2}}=-\mathrm{v}_{\mathrm{f}}{ }^{2} \frac{\partial^{4} \mathrm{D}}{\partial \mathrm{x}_{0}{ }^{4}}-2 \frac{\partial^{2} \mathrm{D}}{\partial \mathrm{T}_{0} \partial \mathrm{T}_{1}}-2 \mathrm{v} \frac{\partial^{2} \mathrm{D}}{\partial \mathrm{x}_{0} \partial \mathrm{T}_{1}} \\
\frac{\partial^{2} \mathrm{E}_{1}}{\partial \mathrm{T}_{0}{ }^{2}}+2 \mathrm{v} \frac{\partial^{2} \mathrm{E}_{1}}{\partial \mathrm{x}_{0} \partial \mathrm{T}_{0}}+5\left(1-\mathrm{v}^{2}\right) \frac{\partial^{2} \mathrm{E}_{1}}{\partial \mathrm{x}_{0}{ }^{2}}=2 \mathrm{v} \frac{\sqrt{1-\mathrm{v}^{2}}}{\mathrm{v}_{\mathrm{f}}} \frac{\partial \mathrm{G}_{1}}{\partial \mathrm{T}_{0}}+2 \frac{\left(1-\mathrm{v}^{2}\right)^{3 / 2}}{\mathrm{v}_{\mathrm{f}}} \frac{\partial \mathrm{G}_{1}}{\partial \mathrm{x}_{0}}
\end{gathered}
$$

Substituting the solutions obtained to the expansion, one has the approximate boundary layer solution

$$
\mathrm{y}=\mathrm{D}\left(\mathrm{x}_{0}, \mathrm{~T}_{0}, \mathrm{~T}_{1}\right)+\varepsilon\left(\mathrm{E}_{1}\left(\mathrm{x}_{0}, \mathrm{~T}_{0}, \mathrm{~T}_{1}\right) \mathrm{e}^{-\frac{\sqrt{1-\mathrm{v}^{2}}}{\mathrm{v}_{\mathrm{f}}} \mathrm{x}_{1}}+\mathrm{F}_{1}\left(\mathrm{x}_{0}, \mathrm{~T}_{0}, \mathrm{~T}_{1}\right)\right)+\ldots \ldots
$$

The above solution contains the inner expansion at the left hand side and the outer expansion. One may now calculate the outer expansion and the right hand side boundary layer solution by defining

$$
\mathrm{x}_{2}=\frac{1-\mathrm{x}}{\sqrt{\varepsilon}}
$$


A similar calculation with only inserting $\mathrm{x}_{2}$ instead of $\mathrm{x}_{1}$ makes some sign changes in the equations. Finally the solution for this case is

$$
y=D\left(x_{0}, T_{0}, T_{1}\right)+\varepsilon\left(E_{2}\left(x_{0}, T_{0}, T_{1}\right) e^{-\frac{\sqrt{1-v^{2}}}{v_{f}} x_{2}}+F_{2}\left(x_{0}, T_{0}, T_{1}\right)\right)+\ldots .
$$

To obtain the composite expansion valid for all parts of the domain, one has to add solutions (23) and (25) and subtract the outer solution which is common. Hence the final solution is

$\mathrm{y}=\mathrm{D}\left(\mathrm{x}_{0}, \mathrm{~T}_{0}, \mathrm{~T}_{1}\right)+\varepsilon\left(\mathrm{E}_{1}\left(\mathrm{x}_{0}, \mathrm{~T}_{0}, \mathrm{~T}_{1}\right) \mathrm{e}^{-\frac{\sqrt{1-\mathrm{v}^{2}}}{\mathrm{v}_{\mathrm{f}}} x_{1}}+\mathrm{E}_{2}\left(\mathrm{x}_{0}, \mathrm{~T}_{0}, \mathrm{~T}_{1}\right) \mathrm{e}^{-\frac{\sqrt{1-\mathrm{v}^{2}}}{\mathrm{v}_{\mathrm{f}}} \mathrm{x}_{2}}+\mathrm{F}_{1}\left(\mathrm{x}_{0}, \mathrm{~T}_{0}, \mathrm{~T}_{1}\right)\right)+\ldots$

\section{BOUNDARY CONDITIONS}

In this section, the boundary conditions will be applied to the composite expansion (26). One has to solve equation (17) first subject to the boundary conditions

$$
\mathrm{D}\left(0, \mathrm{~T}_{0}, \mathrm{~T}_{1}\right)=\mathrm{D}\left(1, \mathrm{~T}_{0}, \mathrm{~T}_{1}\right)=0
$$

Since equation (17) is of second order, these two conditions are the only applicable conditions from the set of boundary conditions given in (2). The solution is

$$
D\left(x_{0}, T_{0}, T_{1}\right)=A_{n}\left(T_{1}\right) e^{i \omega_{n} T_{0}} \psi_{n}\left(x_{0}\right)+\bar{A}_{n}\left(T_{1}\right) e^{-i \omega_{n} T_{0}} \bar{\psi}_{n}\left(x_{0}\right)
$$

where

$$
\psi_{n}=C_{n} e^{i \alpha_{n} x_{0}} \operatorname{Sinn} \pi x_{0}, \quad \omega_{n}=n \pi\left(1-v^{2}\right), \quad \alpha_{n}=n \pi v
$$

Substituting solution (28) into (21), the solvability condition requires

$$
\frac{\partial \mathrm{A}_{\mathrm{n}}}{\partial \mathrm{T}_{1}}+\mathrm{k}_{1} \mathrm{~A}_{\mathrm{n}}=0
$$

where

$$
k_{1}=-\frac{i}{2} v_{f}^{2} n^{3} \pi^{3}\left(v^{4}+6 v^{2}+1\right)
$$

The solution of (30) is

$$
A_{n}=A_{0} e^{-k_{1} T_{1}}
$$

Inserting (32) into (28) and defining $\mathrm{A}_{0}=\frac{1}{2} a \mathrm{e}^{\mathrm{i} \theta}$, one finally obtains 
$D\left(x_{0}, T_{0}, T_{1}\right)=C_{n} \operatorname{Cos}\left\{n \pi\left(1-v^{2}\right) T_{0}+\frac{1}{2} n^{3} \pi^{3}\left(v^{4}+6 v^{2}+1\right) T_{1}+n \pi v x_{0}+\theta\right\} \operatorname{Sinn} \pi x_{0}$

The right hand side of (21) is zero and the left hand side may now be chosen as

$$
\mathrm{F}_{1}\left(\mathrm{x}_{0}, \mathrm{~T}_{0}, \mathrm{~T}_{1}\right)=0
$$

Since the composite expansion is constructed upto order $\varepsilon$, there is no need to determine $\mathrm{G}_{1}$ from (22)

One has to determine $E_{1}$ now. A solution satisfying equation (19) is

$$
E_{1}=C_{n} \alpha_{1} \operatorname{Sin}\left[n \pi\left(1-v^{2}\right) T_{0}+\frac{1}{2} n^{3} \pi^{3}\left(v^{4}+6 v^{2}+1\right) T_{1}-n \pi v x_{0}+\theta_{1}\right]
$$

Similarly

$$
E_{2}=C_{n} \alpha_{2} \operatorname{Sin}\left[n \pi\left(1-v^{2}\right) T_{0}+\frac{1}{2} n^{3} \pi^{3}\left(v^{4}+6 v^{2}+1\right) T_{1}-n \pi v x_{0}+\theta_{2}\right]
$$

Substituting (33)-(36) into (26) and imposing the boundary conditions, one sees that the solution has an order $(\varepsilon)$ error for conditions $y(0, t)=y(1, t)=0$.

For the remaining boundary conditions, to eliminate the error at the first order of approximation, $\alpha_{1}, \theta_{1}, \alpha_{2}$ and $\theta_{2}$ in (35) and (36) are selected as

$$
\begin{aligned}
& \alpha_{1}=\frac{2 \mathrm{v}_{\mathrm{f}}{ }^{2} \mathrm{n}^{2} \pi^{2} \mathrm{v}}{1-\mathrm{v}^{2}} \quad \theta_{1}=\theta \\
& \alpha_{2}=\frac{2 \mathrm{v}_{\mathrm{f}}{ }^{2} \mathrm{n}^{2} \pi^{2} \mathrm{v}}{1-\mathrm{v}^{2}} \cos \mathrm{n} \pi \quad \theta_{2}=\theta+2 \mathrm{n} \pi \mathrm{v}
\end{aligned}
$$

Hence the solution in terms of original variables is

$$
\begin{aligned}
y(x, t)= & C_{n}\left\{\operatorname{Cos}\left[\left(n \pi\left(1-v^{2}\right)+\varepsilon \frac{1}{2} n^{3} \pi^{3}\left(v^{4}+6 v^{2}+1\right)\right) t+n \pi v x+\theta\right\} \operatorname{Sinn} \pi x\right. \\
& +\varepsilon\left(\frac{2 v_{f}{ }^{2} n^{2} \pi^{2} v}{1-v^{2}} \operatorname{Sin}\left[\left(n \pi\left(1-v^{2}\right)+\varepsilon \frac{1}{2} n^{3} \pi^{3}\left(v^{4}+6 v^{2}+1\right)\right) t-n \pi v x+\theta\right] e^{-\frac{\sqrt{1-v^{2}} x}{v_{f}} \sqrt{\varepsilon}}\right. \\
& +\frac{2 v_{f}^{2} n^{2} \pi^{2} v}{1-v^{2}} \operatorname{Cos} n \pi \operatorname{Sin}\left[\left(n \pi\left(1-v^{2}\right)+\varepsilon \frac{1}{2} n^{3} \pi^{3}\left(v^{4}+6 v^{2}+1\right)\right) t\right. \\
& \left.\left.-n \pi v x+2 n \pi v+\theta] e^{-\frac{\sqrt{1-v^{2}}}{v_{f}} \frac{1-x}{\sqrt{\varepsilon}}}\right)+. .\right\}
\end{aligned}
$$


The above approximate solution has an $\mathrm{O}(\varepsilon)$ error for boundary conditions $y(0, t)=y(1, t)=0$ and an $O(\sqrt{\varepsilon})$ error for boundary conditions $y^{\prime \prime}(0, t)=y^{\prime \prime}(1, t)=0$.

\section{CONCLUDING REMARKS}

An approximate boundary layer solution is presented for an axially moving beam with small flexural stiffness. The method of multiple scales is applied to the problem and the composite expansion including two inner solutions and one outer solution is found.

Comparing the solutions with those of Pellicano and Zirilli [5], one may note that both solutions yield the same error for boundary conditions $\mathrm{y}(0, \mathrm{t})=\mathrm{y}(1, \mathrm{t})=0$, namely $\mathrm{O}(\varepsilon)$ error. While multiple scales yield an $O(\sqrt{\varepsilon})$ error for boundary conditions $y^{\prime \prime}(0, t)=y^{\prime \prime}(1, t)=0$, their solution satisfies the boundary conditions exactly. However contrary to their modified Lindstedt Poincare method, using the method of multiple scales, a single expansion is sufficient and requires no matching between the expansions. This advantage brings another disadvantage: constructing the solutions at each order of $\varepsilon$ is not as straightforward in the method of multiple scales as in the method of matched expansions and requires some experience.

\section{ACKNOWLEDGMENT}

This work is supported by the Scientific and Technical Research Council of Turkey TÜBITAK under project no: MISAG-119.

\section{REFERENCES}

1. A. G. Ulsoy, C.D. Mote Jr. and R. Szymani, Principal developments in band saw vibration and stability research, Holz als Roh-und Werkstoff 36, 273-280, 1978.

2. J.A. Wickert and C.D.Mote Jr., Current research on the vibration and stability of axially moving materials, Shock and Vibration Digest 20(5), 3-13,1988.

3. J.A. Wickert and C.D.Mote Jr., Classical vibration analysis of axially moving continua, ASME, Journal of Applied Mechanics 57, 738-744, 1990.

4. J.A. Wickert, Non-linear vibration of a traveling tensioned beam, International Journal of Non-linear Mechanics 27, 503-517, 1992.

5. F. Pellicano and F. Zirilli, Boundary layers and non-linear vibrations in an axially moving beam, International Journal of Nonlinear Mechanics 33(4), 691-711, 1998

6. H. R. Öz, M. Pakdemirli and E. Özkaya, Transition behavior from string to beam for an axially accelerating material, Journal of Sound and Vibration 215(3), 571-576, 1998.

7. A. H. Nayfeh, Introduction to Perturbation Techniques, Wiley, New - York, 1981 